TABLE DR1. ELEMENT OXIDE AND FELDSPAR COMPOSITIONS FOR AUTHIGENIC K-FELDSPAR (P1C \& P2 $\mathrm{c}$ )

\begin{tabular}{|c|c|c|c|c|c|c|c|c|c|c|c|c|c|c|c|c|}
\hline & & \multirow[b]{2}{*}{$\mathrm{n}$} & \multicolumn{8}{|c|}{ Wt. \% Oxide (Arithmetic mean) } & \multicolumn{6}{|c|}{ Molecular composition (\%) } \\
\hline & & & $\mathrm{SiO}_{2}$ & $\mathrm{TiO}_{2}$ & $\mathrm{Al}_{2} \mathrm{O}_{3}$ & $\mathrm{FeO}$ & $\mathrm{CaO}$ & $\mathrm{Na}_{2} \mathrm{O}$ & $\mathrm{K}_{2} \mathrm{O}$ & $\mathrm{BaO}$ & Total & Or & $A b$ & $\mathrm{Cn}$ & An & Total \\
\hline \multirow[t]{2}{*}{ An-t-Sron } & $\mathrm{P} 1_{\mathrm{c}}$ & 5 & 64.91 & 0.12 & 17.96 & 0.04 & 0.05 & 0.04 & 16.46 & 0.00 & 99.56 & 99.50 & 0.21 & 0.00 & 0.28 & 100 \\
\hline & $\mathrm{P} 2_{\mathrm{c}}$ & 5 & 63.82 & 0.16 & 17.40 & 0.34 & 0.17 & 0.08 & 15.69 & 0.08 & 97.75 & 97.95 & 0.49 & 0.51 & 1.05 & 100 \\
\hline \multirow[t]{2}{*}{ Corry Point } & $\mathrm{P} 1_{\mathrm{c}}$ & 5 & 65.52 & 0.10 & 18.15 & 0.16 & 0.10 & 0.05 & 16.84 & 0.00 & 100.92 & 99.11 & 0.30 & 0.00 & 0.59 & 100 \\
\hline & $\mathrm{P} 2_{\mathrm{c}}$ & 5 & 63.99 & 0.17 & 17.90 & 0.05 & 0.04 & 0.05 & 16.02 & 0.11 & 98.33 & 98.78 & 0.31 & 0.68 & 0.23 & 100 \\
\hline \multirow[t]{2}{*}{ Ord } & $\mathrm{P} 1_{\mathrm{c}}$ & 5 & 64.81 & 0.13 & 19.97 & 0.05 & 0.17 & 0.02 & 16.41 & 0.00 & 101.55 & 98.89 & 0.10 & 0.00 & 1.01 & 100 \\
\hline & $\mathrm{P} 2_{\mathrm{c}}$ & 5 & 65.19 & 0.00 & 17.97 & 0.16 & 0.07 & 0.15 & 16.55 & 0.07 & 100.16 & 98.29 & 0.90 & 0.41 & 0.40 & 100 \\
\hline
\end{tabular}

Or - orthoclase; Ab - albite; $\mathrm{Cn}$ - celsian; An - anorthite (data normalized using 32 oxygens) 
Table DR2. Ar-Ar DATA FOR AUTHIGENIC K-FELDSPAR AN-T-SRON

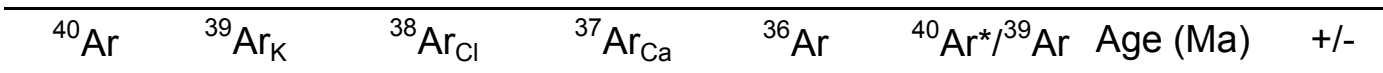

An-t-Sron: $\mathbf{P} \mathbf{2}_{\mathbf{C}}$

0.213220 .00463

0.169750 .00385

0.365390 .00883

0.278930 .00626

0.276820 .00619

0.202090 .00445

$0.2026 \quad 0.00476$

$0.28893 \quad 0.00667$

0.244320 .00542

0.251950 .00621

0.0001

$0.0057 \quad 0.00027$

43.07422

$438.6 \quad 15.1$

0.00014

0.00584

0.0003

41.30450

422.6

19.1

0.00021

$0.00447 \quad 0.00037$

41.88790

0.00018

$0.00568 \quad 0.0003$

43.16367

0.00015

$0.0052 \quad 0.00038$

43.30955

0.00011

0.00462

0.0002

42.93044

0.00011

0.00464

0.00033

40.27132

0.0002

0.00572

0.00034

43.81579

0.00011

0.00434

0.00016

43.19193

0.00021

0.00457

0.00048

44.38366

427.9

439.5

440.8

437.3

413.1

8.6

11.3

11.5

An-t-Sron: $\mathbf{P} \mathbf{1}_{\mathbf{C}}$

$0.18954 \quad 0.00391$

0.267490 .00546

0.00018

0.0056

0.00014

45.00893

445.3

16.0

11.1

0.274110 .00539

0.00007

0.00463

0.0002

46.37573

439.7

14.5

$450.4 \quad 14.4$

0.280030 .00586

0.368370 .00811

0.188310 .00376

0.0001

0.00425

0.00009

48.23645

$456.1 \quad 17.6$

0.00016

0.0052

0.00023

0.00019

0.00475

0.00027

45.76293

0.00019

0.00526

0.0002

44.36327

$468.2 \quad 12.5$

$0.2529 \quad 0.00533$

0.00023

0.00551

0.00032

47.26564

$484.7 \quad 12.9$

$0.30533 \quad 0.00649$

0.00015

0.00533

0.00033

45.75761

462.8

11.7

450.3

8.7

0.252790 .00549

0.00016

0.00574

0.00039

46.05375

476.1

18.8

0.363320 .0082

0.00017

0.00496

0.00047

47.00430

462.7

13.3

$0.45946 \quad 0.00987$

0.285530 .00568

0.00021

0.00475

0.00049

46.21019

465.4

11.3

$473.8 \quad 13.3$

$\begin{array}{lll}0.3297 & 0.00665\end{array}$

0.00015

0.0048

0.00016

48.82547

466.8

10.1

0.00018

0.0055

0.00029

48.53051

489.9

7.7

$\begin{array}{lll}0.3982 & 0.00841\end{array}$

0.00017

0.00452

0.00019

48.02949

487.3

12.2

$482.9 \quad 10.4$

$0.25964 \quad 0.00559$

0.00016

0.00457

46.21455

466.8

8.6

$0.27003 \quad 0.00558$

0.00542

0.00025

44.88144

454.9

13.1

$0.27066 \quad 0.00594$

0.00018

0.00023

46.89556

472.9

12.5

$0.25942 \quad 0.00557$

0.00014

0.00531

0.00053

47.62449

$479.3 \quad 17.4$

0.00013

0.00545

0.00027

48.86898

490.3

14.0

$0.22497 \quad 0.00451$

0.00009

0.00468

0.00009

47.61015

479.2

17.1

$0.24776 \quad 0.00513$

0.00016

0.00475

0.00015

46.80502

472.1

15.2

$0.24651 \quad 0.00506$

0.00017

0.00462

0.00029

46.83258

472.3

16.2

0.199020 .00404

0.00008

0.00417

0.00024

46.74636

471.5

19.2

$\begin{array}{lll}0.25631 & 0.00524\end{array}$

0.00521

0.00029

48.14448

483.9

15.1

0.313450 .0066

0.00019

0.00504

0.0004

46.81861

$472.2 \quad 12.5$

\section{Mean blank}

$\begin{array}{llllll}0.01938 & 0.00016 & 0.00008 & 0.00451 & 0.000231 & (\mathrm{n}=57)\end{array}$

Quoted Ar-Ar errors are $2 \sigma\left(\times 10^{12} \mathrm{~cm}^{3} \mathrm{STP}\right)$ and include a $0.5 \%$ error assigned to the $\mathrm{J}$ value. Reactor induced correction factors used were: $\left({ }^{39} \mathrm{Ar} r^{37} \mathrm{Ar}\right)_{\mathrm{Ca}}=0.00065,\left({ }^{38} \mathrm{Ar}{ }^{37} \mathrm{Ar}\right)_{\mathrm{Ca}}=0.000264,\left({ }^{40} \mathrm{Ar}{ }^{39} \mathrm{Ar}\right)_{\mathrm{K}}=0.0085$. 
Table DR3. Ar-Ar DATA FOR AUTHIGENIC K-FELDSPAR SKIAG BRIDGE

\begin{tabular}{|c|c|c|c|c|c|c|c|}
\hline${ }^{40} \mathrm{Ar}$ & ${ }^{39} \mathrm{Ar}_{\mathrm{K}}$ & ${ }^{38} \mathrm{Ar}_{\mathrm{Cl}}$ & ${ }^{37} \mathrm{Ar}_{\mathrm{Ca}}$ & ${ }^{36} \mathrm{Ar}$ & ${ }^{40} \mathrm{Ar} * /{ }^{39} \mathrm{Ar}$ & Age (Ma) & $+/-$ \\
\hline \multicolumn{8}{|c|}{ Skiag Bridge: $\mathrm{P} 2_{\mathrm{C}}$} \\
\hline 0.26244 & 0.01272 & 0.0003 & 0.00352 & 0.00032 & 20.21074 & 448.9 & 12.0 \\
\hline 0.1317 & 0.00631 & 0.0001 & 0.00284 & 0.00024 & 19.83416 & 441.5 & 24.4 \\
\hline 0.08579 & 0.00414 & 0.00015 & 0.00348 & 0.00023 & 18.65152 & 418.0 & 37.5 \\
\hline 0.19792 & 0.00995 & 0.00019 & 0.00336 & 0.00028 & 19.03883 & 425.8 & 15.2 \\
\hline 0.14023 & 0.00665 & 0.00016 & 0.00268 & 0.00037 & 19.83115 & 441.5 & 22.7 \\
\hline 0.19038 & 0.0095 & 0.00018 & 0.0032 & 0.00019 & 19.05763 & 426.1 & 16.0 \\
\hline 0.12902 & 0.00593 & 0.00014 & 0.0048 & 0.00026 & 20.20862 & 448.9 & 25.3 \\
\hline 0.21517 & 0.01075 & 0.00021 & 0.00305 & 0.00034 & 19.18538 & 428.7 & 14.0 \\
\hline 0.08263 & 0.00385 & 0.0001 & 0.00285 & 0.00022 & 19.34193 & 431.8 & 39.9 \\
\hline 0.19918 & 0.00939 & 0.00022 & 0.0031 & 0.00021 & 20.36043 & 451.9 & 16.7 \\
\hline 0.22422 & 0.01155 & 0.0003 & 0.00335 & 0.00024 & 18.59641 & 416.9 & 13.1 \\
\hline 0.12147 & 0.00625 & 0.00011 & 0.00475 & 0.00016 & 17.91361 & 403.2 & 24.5 \\
\hline 0.25244 & 0.0133 & 0.00021 & 0.00281 & 0.00025 & 18.21128 & 409.2 & 11.4 \\
\hline 0.17249 & 0.00839 & 0.00022 & 0.00311 & 0.00029 & 19.39431 & 432.8 & 18.2 \\
\hline 0.13132 & 0.00592 & 0.00007 & 0.00316 & 0.00023 & 20.55834 & 455.8 & 25.7 \\
\hline 0.11365 & 0.00534 & 0.00014 & 0.00462 & 0.0002 & 19.65448 & 438.0 & 28.3 \\
\hline 0.10354 & 0.00493 & 0.00022 & 0.0046 & 0.0003 & 19.22571 & 429.5 & 30.8 \\
\hline 0.08111 & 0.00385 & 0.00014 & 0.00294 & 0.00024 & 19.60942 & 437.1 & 40.8 \\
\hline 0.30636 & 0.01467 & 0.00032 & 0.00333 & 0.00028 & 20.36539 & 452.0 & 10.3 \\
\hline 0.25054 & 0.01272 & 0.00022 & 0.0031 & 0.00025 & 19.07362 & 426.4 & 12.3 \\
\hline 0.23637 & 0.01251 & 0.00022 & 0.00325 & 0.00024 & 18.42180 & 413.4 & 12.4 \\
\hline 0.29951 & 0.01595 & 0.00017 & 0.00311 & 0.00023 & 18.40708 & 413.1 & 9.8 \\
\hline 0.26546 & 0.01361 & 0.00031 & 0.00333 & 0.00026 & 19.10990 & 427.2 & 11.6 \\
\hline 0.21781 & 0.01061 & 0.00026 & 0.00342 & 0.00025 & 20.05314 & 445.8 & 14.4 \\
\hline 0.30309 & 0.0154 & 0.00036 & 0.00339 & 0.00025 & 19.29404 & 430.8 & 9.9 \\
\hline 0.31468 & 0.01551 & 0.00032 & 0.00315 & 0.00024 & 19.90255 & 442.9 & 10.0 \\
\hline 0.28344 & 0.01432 & 0.00017 & 0.003 & 0.00029 & 19.36667 & 432.3 & 10.7 \\
\hline 0.25332 & 0.01255 & 0.00018 & 0.00369 & 0.00027 & 19.77389 & 440.3 & 12.1 \\
\hline 0.24351 & 0.0126 & 0.00024 & 0.00364 & 0.00036 & 18.79613 & 420.9 & 12.1 \\
\hline 0.2538 & 0.01317 & 0.00022 & 0.0032 & 0.00023 & 18.65939 & 418.2 & 11.5 \\
\hline 0.22735 & 0.01075 & 0.00035 & 0.00339 & 0.00029 & 20.36171 & 451.9 & 14.0 \\
\hline 0.26139 & 0.01364 & 0.00031 & 0.00435 & 0.00024 & 18.48856 & 414.8 & 11.3 \\
\hline \multicolumn{8}{|c|}{ Skiag Brtidge: $\mathrm{P} 1_{\mathrm{C}}$} \\
\hline 0.14068 & 0.00618 & 0.00022 & 0.00328 & 0.00028 & 21.99316 & 483.7 & 24.7 \\
\hline 0.14505 & 0.00633 & 0.00013 & 0.0028 & 0.00024 & 21.97521 & 483.3 & 23.9 \\
\hline 0.34915 & 0.01643 & 0.00034 & 0.00321 & 0.00027 & 20.72144 & 459.0 & 9.1 \\
\hline 0.23388 & 0.0107 & 0.00025 & 0.00297 & 0.00027 & 20.97258 & 463.9 & 13.9 \\
\hline 0.28098 & 0.01305 & 0.00024 & 0.00343 & 0.00033 & 20.80348 & 460.6 & 12.3 \\
\hline 0.14067 & 0.00634 & 0.00011 & 0.00279 & 0.00026 & 21.36885 & 471.6 & 23.7 \\
\hline 0.17561 & 0.0076 & 0.00021 & 0.00414 & 0.00017 & 22.14526 & 486.6 & 19.6 \\
\hline 0.08088 & 0.00341 & 0.00005 & 0.00409 & 0.00031 & 21.50538 & 474.2 & 44.1 \\
\hline 0.12587 & 0.00585 & 0.00012 & 0.00348 & 0.00026 & 20.67235 & 458.0 & 26.1 \\
\hline 0.14909 & 0.00686 & 0.0001 & 0.0035 & 0.00025 & 21.03485 & 465.1 & 23.1 \\
\hline 0.23908 & 0.0105 & 0.00021 & 0.00364 & 0.00028 & 22.33219 & 490.2 & 14.8 \\
\hline 0.24197 & 0.01145 & 0.00016 & 0.00337 & 0.00024 & 20.70299 & 458.6 & 13.6 \\
\hline 0.17202 & 0.0078 & 0.0001 & 0.00338 & 0.0003 & 21.25756 & 469.4 & 19.4 \\
\hline 0.10646 & 0.00452 & 0.00012 & 0.00315 & 0.00026 & 21.85568 & 481.0 & 32.8 \\
\hline 0.30471 & 0.01412 & 0.00027 & 0.00317 & 0.00036 & 20.98607 & 464.1 & 10.5 \\
\hline \multicolumn{8}{|c|}{ Mean blank } \\
\hline 0.01186 & 0.0002 & 0.00005 & 0.00278 & 0.00023 & $(n=62)$ & & \\
\hline
\end{tabular}

Quoted Ar-Ar errors are $2 \sigma\left(\times 10^{12} \mathrm{~cm}^{3}\right.$ STP $)$ and include a $0.5 \%$ error assigned to the $\mathrm{J}$ value. Reactor induced correction factors used were: $\left({ }^{39} \mathrm{Ar} /{ }^{37} \mathrm{Ar}\right)_{\mathrm{Ca}}=0.00065,\left({ }^{38} \mathrm{Ar} /{ }^{37} \mathrm{Ar}\right)_{\mathrm{Ca}}=0.000264,\left({ }^{40} \mathrm{Ar} /{ }^{39} \mathrm{Ar}\right)_{\mathrm{K}}=0.0085$. 